\title{
Blank-comparison matching-to-sample reveals a false positive symmetry test in a capuchin monkey
}

\author{
Ana L. F. Brino ${ }^{1}$, Rodolfo S. Campos ${ }^{1}$, Olavo F. Galvão ${ }^{1}$, and William J. McIlvane ${ }^{2}$ \\ 1. Universidade Federal do Pará, Belém, PA, Brazil \\ 2. University of Massachusetts Medical School, Worcester, MA, United States
}

\begin{abstract}
A positive symmetry test result was obtained with a capuchin monkey that had previously exhibited virtually errorless AB and BA arbitrary matching-to-sample (MTS) with different stimuli. The symmetry test (BA) followed the acquisition of a new $\mathrm{AB}$ relation. It seemed possible, however, that the positive result could have occurred through the exclusion of previously defined comparison stimuli and not because the new $\mathrm{AB}$ and BA relations had the property of symmetry. To assess this possibility, a blank-comparison MTS procedure was implemented that permitted the separate assessment of select and reject (i.e., exclusion) control with both baseline and BA matching relations. In this assessment, the monkey did not exhibit reliable BA matching when exclusion was not possible, thus showing that the symmetry result was a false positive. However, the study demonstrated the feasibility of using a blank comparison MTS procedure with capuchins. The present results may set the stage for more successful methodology for establishing desired forms of relational stimulus control in capuchins and ultimately improving the assessment of relational learning capacity in that species, other nonhuman species, and nonverbal humans. Keywords: arbitrary matching-to-sample, exclusion, symmetry, Sapajus sp.
\end{abstract}

Received 18 June 2013; received in revised form 06 February 2014; accepted 10 February 2014. Available online 27 June 2014.

\section{Introduction}

Relational learning, a kind of generalized performance or "inferential reasoning" (Aust, Range, Steurer, \& Huber, 2008), is considered a foundational skill for typical human development (McIlvane, Dube, Serna, Lionello-DeNolf, Barros, \& Galvão, 2011). For example, generalized identity matching-to-sample (MTS; Serna, Dube, \& Mcllvane, 1997), learning by exclusion (Wilkinson, Dube, \& McIlvane, 1996), and fast mapping phenomena (Kaminski, Call, \& Fischer, 2004) have been considered important processes responsible for errorless learning and emergent enlargement of exclusive one-to-one relationships as in naming. Stimulus equivalence (Sidman, 1994) has been considered a fundamental process of that same type, involving arbitrary relational learning that is necessary for the emergence of new adaptive repertoires.

Emergent relational repertoires that indicate stimulus equivalence in animals have been

Ana Leda de Faria Brino, Rodolfo da Silva Campos, and Olavo de Faria Galvão, Universidade Federal do Pará. William Jay McIlvane, University of Massachusetts Medical School. Correspondence regarding to article should be directed to: Ana Leda F. Brino, Núcleo de Teoria e Pesquisa do Comportamento, Universidade Federal do Pará. Rua Augusto Corrêa, n¹, Guamá, Belém, PA, CEP 66075-110, Brasil. E-mail: abrino@gmail.com demonstrated. Vaughan (1988) and Frank and Wasserman (2005) reported functional class formation and associative symmetry, respectively, in pigeons. Schusterman and Kastak (1993) and Kastak, Schusterman, and Kastak (2001) studied sea lions and reported emergent symmetric, transitive, and equivalence relations (Sidman \& Tailby, 1982). However, the necessary conditions to obtain the emergence of derived relations in nonhumans have not been established (Sidman, 1994; Galvão et al., 2005). Knowledge of the variables responsible for intra- and inter-subject baseline performance variability has been suggested to be a possible source for predicting derived emergent relations (Galvão et al., 2005). Extant reports of derived repertoires involve special sets of conditions with various species, but no analysis of the variables that could explain both successes and failures has been performed. Indeed, we lack information about how relational repertoires could be built under laboratory conditions.

Sidman, Rauzin, Lazar, Cunningham, Tailby, and Carrigan (1982) suggested some important conditions that might favor emergent symmetry in nonhumans, among them multiple exemplar training, variation of stimulus location, and generalized identity matching as a prerequisite. In addition to prerequisites reported by Sidman et al. (1982), later studies with pigeons (Frank \& Wasserman, 2005) and sea lions (Kastak et al., 
2001) suggested that establishing both select and reject controlling relations among elements within arbitrary MTS baseline may be critical for equivalence class formation (Lionello-DeNolf, 2009).

In usual discrete-trial simple or conditional discrimination tasks, the subject is required to choose one of a number of simultaneously presented stimuli. In conditional discrimination training, the control over choice responses by the relation between the sample and positive stimulus is called select control, and the control by the relation between the sample and negative stimulus is called reject control. Specifically, select control refers to the Sample- $\mathrm{S}^{+}$relation, and reject control refers to the Sample-S- relation. Both types of control may occur concurrently in the same manner that different dimensions of an event can control the behavior (Johnson \& Sidman, 1993). Recognizing this diversity in stimulus control within a subject across trials, Ray (1969) created the term stimulus control topography.

To test hypotheses of the importance of select and reject control in producing emergent performance and stimulus class formation, we require a reliable methodology for measuring and perhaps promoting the topographies of stimulus control (c.f., McIlvane \& Dube, 2003). A small amount of prior work addressed this needed methodological development. For example, two studies showed that capuchin monkeys can acquire arbitrary stimulus-stimulus relations consistent with the development of select and reject control (c.f., Brino, Assumpção, Campos, Galvão, \& McIlvane, 2010; Brino, Galvão, Barros, Goulart, \& McIlvane, 2012). To date, however, no study has used the methodology to systematically assess whether necessary select or reject relations have been established within a stimulus equivalence framework with nonhumans. Notably, such methods have been used with humans, and the results have been consistent with past hypotheses about the importance of establishing select and reject relations to support equivalence class formation (e.g., Stromer \& Osborne, 1982; de Rose, Hidalgo, \& Vasconcellos, 2013).

The monkey that served as the subject in the present study had an extensive history of simple and conditional discrimination learning. In studies reported by Brino et al. (2010), Brino et al. (in press), and Campos, Brino, and Galvão (2013), the capuchin acquired generalized identity matching (IDMTS) and multiple arbitrary matching (ARBMTS) baselines, demonstrating that reject control could be developed via blank comparison (mask) procedures (McIlvane, Kledaras, Munson, King, de Rose, \& Stoddard, 1987). The present study exploited this unusually well-developed baseline in an effort to analyze stimulus control topographies, supporting a positive outcome on a test for emergent symmetry.

Previous direct training established four pairs (A1B1 to A4B4) of arbitrary stimulus relations and their symmetric counterparts (B1A1 to B4A4). Another pair (A5B5) was established to set the stage for a test of emergent symmetry (i.e., B5A5 immediately present in the test without prior direct discrimination training). This paper reports these symmetry test results and analyzes possible select and reject relations that helped clarify the meaning of the test outcome. As in the earlier studies with this subject, the select/reject stimulus control analysis used the blank comparison procedure.

\section{Methods}

\section{Subject}

Raul (M14; Figure 1) was an adult male capuchin monkey (Sapajus sp.; see Alfaro, Silva, \& Rylands, 2012, for an explanation of the new nomenclature for robust capuchins, formerly part of the Cebus genus). As noted, Raul had an extensive MTS history, including (1) generalized IDMTS (Galvão et al., 2005; Brino, Galvão, \& Barros, 2009; Brino et al., in press), (2) ARBMTS with (a) positive outcomes on tests of restricted control and (b) previous negative outcomes on symmetry tests (Brino, 2007; Brino et al., 2012), and (3) positive outcomes on tests for exclusion (Brino et al., 2010; Brino et al., 2013). Raul was housed in a steel wire cage measuring $2.5 \mathrm{~m}^{3}$ half covered by Monk and Num ceramic tiles. In each corner of the cage, $1.0 \mathrm{~m}$ above the pavement there was a small $\left(0.5 \mathrm{~m}^{3}\right)$ cage with doors that allowed access to the interior and exterior of the cage and to a isolation corridor with a rectangular aperture $(0.05 \mathrm{~m} \times 0.15 \mathrm{~m})$ with supports for attaching a foodtray outside. The enclosure had two levels of wood lath paths that ran around and across the cage and a house with four compartments, with two entrances each. Every day, a small number of toys, paper, wood, or hard-plastic objects were inserted for manipulation. A small meal was delivered in the morning, and a large meal (pellet food for monkeys, fruits, roots, and eggs) was delivered at 3:00 PM. Water was always available. The biotery was supervised by a veterinary-physician and up-to-date with regard to Brazilian and International Standards for the humane care and maintenance of research animals.

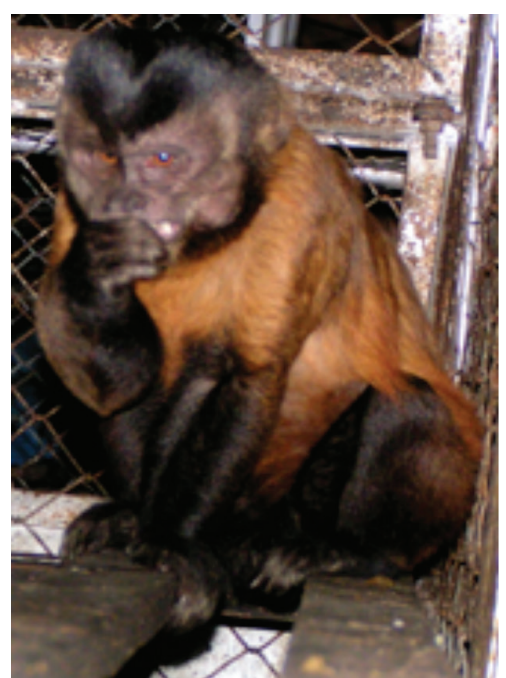

Figure 1. Raul (M14), an adult male capuchin monkey. 


\section{Experimental environment and apparatus}

The experimental chamber (see Figure 2) measured $0.60 \mathrm{~m} \times 0.60 \mathrm{~m} \times 0.60 \mathrm{~m}$. It was equipped with a touchsensitive monitor with access to it through a $0.26 \mathrm{~m} \times$ $0.26 \mathrm{~m}$ opening on one wall, a pellet dispenser (ENV203-190, Med Associates, St. Albans, VT, USA), and receptacles for pellet deliveries above the opening to the monitor. The sessions were controlled by a computer that ran EAM 4.0.04 software designed by Drauzio Capobianco for discrimination training, pellet dispensing, and data acquisition. The sessions were recorded and could be observed in real time from an adjacent room. Sessions were run from Monday to Friday at 11:00 AM and lasted from 10 to $15 \mathrm{~min}$.

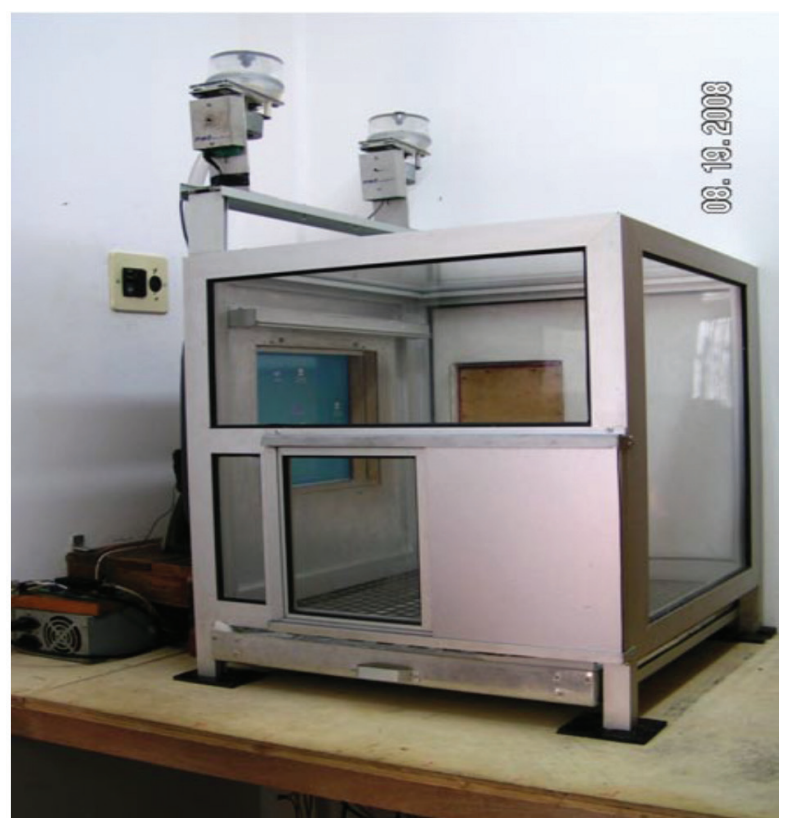

Figure 2. Experimental chamber with touch screen and pellet dispenser.

\section{Stimuli}

Figure 3 shows the stimuli used in the arbitrary matching training and test phases. They were $5 \mathrm{~cm} \times 5$ $\mathrm{cm}$ color images presented in any of nine positions in a $3 \times 3$ matrix on the monitor. The mask used in the blank comparison procedure was a $5 \mathrm{~cm} \times 5 \mathrm{~cm}$ white square.
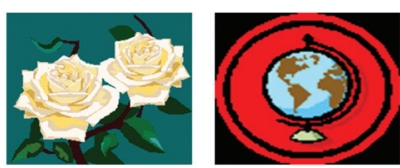

A1-B1/B1-A1
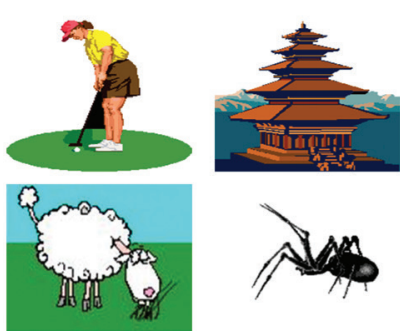

A2-B2 /B2-A2

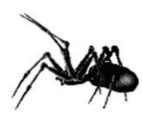

A5-B5 /B5-A5

Figure 3. Sample and comparison stimuli from baseline and test relations.
General procedure for training and testing phases

A previously acquired ARBMTS baseline had displayed five comparisons in every trial. It consisted of five $\mathrm{AB}$ and four BA matching relations. A delayed arbitrary MTS procedure was used. Trials began with sample presentation. After five touches to the sample, it was removed, initiating a 1-s delay before two or three comparisons were displayed simultaneously. In the initial sessions, one touch to a comparison was required. Beginning in session $n$, two touches to the $\mathrm{S}^{+}$ were required to avoid casual touches being recorded as choices. No feedback was given to the first touch in the correct or incorrect comparisons. If the selection was correct, then a pellet was delivered, and a 6-s intertrial interval (ITI) ensued. Incorrect responses were followed only by a 6-s ITI. The sessions had 30-54 trials, depending on the number of trial types in the session, to balance the number of trials of each trial type. A correction procedure was used during training to promote acquisition and maintain a high density of reinforced trials. Following an error, the same trial was repeated until a correct selection was made. Such selections were not counted as correct responses.

\section{Phase 1. Preparation for symmetry test}

Three AB baseline relations (A1B1, A2B2, A5B5) and two BA baseline relations (B1A1, B2A2) with the highest accuracy were reviewed in a three-choice MTS procedure. Relation B5A5 was reserved for the symmetry test. Sessions with and without blankcomparison trials were presented in alternation. To complete this phase, $90 \%$ accuracy was required for three consecutive sessions without the mask and three consecutive sessions with the mask.

\section{Phase 2. Symmetry test}

Test sessions were composed of eight threecomparison B5A5 symmetry trials interspersed within 24 AB (A1B1, A2B2, A5B5) and 16 BA (B1A1, B2A2) baseline trials. All were run with programmed reinforcement for correct choices. No blank comparison or correction procedures were used.

\section{Phase 3. Assessment of controlling relations}

After three symmetry test sessions, a mask or a new stimulus was substituted for one of the comparisons in some of the trials to evaluate select and reject controlling relations for the same three pairs of $\mathrm{AB}$ and $\mathrm{BA}$ relations that had been presented during the symmetry test. The sessions had 54 trials, 45 baseline A-B and B-A and nine B5-A5 (test relation). The trials could have two or three comparisons, some with the mask, some without it, and some with a new stimulus. Table 1 shows examples of each trial and the proportions of two vs. three comparison trials and mask vs. no mask trials.

In total, there were nine trial types for each relation presented in the symmetry test sessions in Phase 3. For purposes of illustration, Table 1 presents all of the trial 
types corresponding to the B5A5 relation. It shows that in trials that tested reject relations, the mask was substituted for comparison A5. In trials that tested select relations and/or novelty preference, the mask or a new stimulus was substituted for stimuli that had been the $\mathrm{S}^{-}$in relation to the sample $\mathrm{B} 5$.

Table 1. Performance on the nine B5-A5 symmetry-test trials. The sample was always B5. Correct choice $\left(\mathrm{S}^{+}\right)$could be stimulus A5 or MK. Incorrect choices $\left(\mathrm{S}^{-}\right)$could be stimulus A1, A2, New, or MK; a given trial could have one or two S-. Control relations could be S, selection; R, rejection; N, novelty, depending on the choices available. Most right column show Correct (C), and Incorrect (X) responses for each trial.

\begin{tabular}{ccccc}
\hline $\mathbf{S}^{+}$ & $\mathbf{S}^{-}$ & $\mathbf{S}^{-}$ & Control Relation Assessed & Results \\
\hline A5 & A1 & - & S or R & C \\
A5 & A2 & - & S or R & C \\
A5 & New & - & N & C \\
A5 & MK & - & S & X \\
MK & A1 & - & R & C \\
MK & A2 & - & R & C \\
MK & A1 & A2 & R & C \\
A5 & MK & A1 & S & X \\
A5 & A2 & MK & S & X \\
\hline
\end{tabular}

Table 2. Number of correct choices in select and reject trials in response to each relation presented in mask sessions applied in Phase 1 (Preparation for symmetry test).

\begin{tabular}{ccc}
\hline Relation & $\begin{array}{c}\text { Reject-control trials } \\
\text { MK substituting for S }\end{array}$ & $\begin{array}{c}\text { Select-control trials } \\
\text { MK substituting for one S }\end{array}$ \\
\hline A1-B1 & $4 / 5$ & $9 / 10$ \\
B1-A1 & $4 / 5$ & $7 / 10$ \\
A2-B2 & $5 / 5$ & $10 / 10$ \\
B2-A2 & $5 / 5$ & $10 / 10$ \\
A5-B5 & $5 / 5$ & $7 / 10$ \\
\hline
\end{tabular}

\section{Results}

At the conclusion of Phase 1, the baseline was stable with accuracy levels above 90\% correct. In fact, Raul showed perfect performance in the last three baseline sessions without a mask and 97.00\%, 88.88\%, and $100.00 \%$ correct responses (average, $95.29 \%$ ) in the last three sessions with the mask. In sessions in which no mask appeared, performance was virtually perfect (23 or 24 correct in A1B1 trials and no errors with relations $\mathrm{B} 1 \mathrm{~A} 1, \mathrm{~A} 2 \mathrm{~B} 2, \mathrm{~B} 2 \mathrm{~A} 2$, and $\mathrm{A} 5 \mathrm{~B} 5)$. In three sessions that programmed trials with the mask that substituted for a positive comparison stimulus (reject) or negative comparison stimulus (select), Table 2 shows that few errors occurred, with most occurring in the latter trial types.

In Phase 2, in three symmetry test sessions, performance was virtually errorless in the baseline (A1B1/B1A1, A2B2/B2A3, A5B5) and test (B5A5) trials (Figure 4).

In Phase 3, Table 2 shows the probe trials that corresponded to the symmetry test that substituted (1) the mask for the $\mathrm{S}^{+}$(reject probes) or $\mathrm{S}^{-}$(select probes) or (2) the new stimulus for an $\mathrm{S}^{-}$. Raul continued to select the A5 comparison accurately in relation to the B5 sample when the $\mathrm{S}^{-}$was A1, A2, or the new stimulus, apparently excluding those incorrect comparison stimuli. When the mask was available, however, Raul always chose it rather than relating A5 with B5 (i.e., a total absence of the select control that was implied by the positive B5A5 symmetry test result in Phase 2).

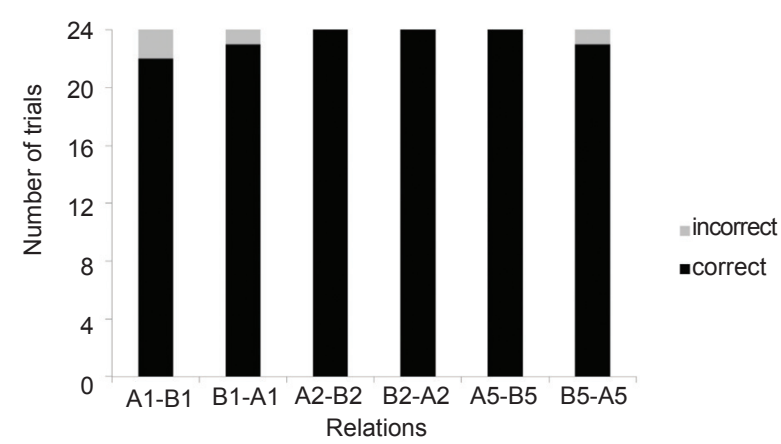

Figure 4. Performance in each relation in three symmetry test sessions.

\section{Discussion}

Had we stopped the experiment after the symmetry test, we would have reported a positive symmetry finding in the capuchin. Indeed, a positive symmetry finding would appear consistent with the fact that the baseline relations, including those evaluated with the blank comparison procedure, were exhibited with fairly high accuracy, including examples of reversed sample and comparison relations A1B1, A2B2, B1A1, and B2A2. Nevertheless, the findings of the symmetry test with blank comparisons (Phase 3 ) revealed that the symmetry finding was a false positive, in which Raul never related the A5 comparison with the B5 sample unless there was a defined $\mathrm{S}^{-}$stimulus to exclude. In discrete trials experiments the use of blank comparison was a stimulus control assessment technique that allows verifying for each trial type what were the stimulus relations controlling choices. Use of this and other direct techniques help us understand the variability reported in the literature and why studies that used such techniques may have succeeded where others had failed (c.f., Carr, Wilkinson, Blackman, \& McIlvane, 2000).

In B5-A5 test trials, errors choosing the mask in trials in which the mask was substituted for an $\mathrm{S}^{-}$could be traced to the following stimulus control topographies: (1) absence of select control (sample- $\mathrm{S}^{+}$relation), (2) rejection of the other available $\mathrm{S}^{-}$, and (3) partial control by the mask as a function of the experimental history of nearly 33\% reinforcement in baseline with three choices and $50 \%$ in test trials, considering that performance was $>90 \%$ correct throughout the phases.

One would be concerned with the fact that in the test trials with the mask the subject always selected the 
mask when it was available, regardless of whether the positive comparison stimulus was absent or present. Would it be possible that the mask had some perceptual features that could attract the subject's attention?

Notably, prior to the symmetry test, the subject received five identity matching sessions with stimulus set A. The history of the mask as the comparison stimulus was far more extensive than that of A5. However, the subject did not choose the mask in four of the 15 baseline trials with the mask that substituted for the $\mathrm{S}^{+}$and correctly chose the $\mathrm{S}^{+}$in all 15 trials with the mask that substituted for the $\mathrm{S}$. This performance clearly indicates that there was no special control by, or any identifiable perceptual preference for, the mask.

Moreover, an interesting parallel was observed with data from humans in similar tasks. Although our monkey showed almost perfect performance in the baseline trials with the mask, indicating the establishment of mixed control (i.e., Sample- $\mathrm{S}^{+}$and Sample-S- relations), and correct performance in the symmetry test without the mask, in the tests with the mask, the subject chose incorrectly in all of the trials in which the Sample- $\mathrm{S}^{+}$control was required, although showing perfect performance in all of the trials in which the Sample-S- control relations were available. These data differ from those presented by Carr et al. (2000), who reported positive symmetry and equivalence tests in subjects with minimal verbal repertoires. Their study indicated that the participants who showed mixed control in baseline relations were those who chose correctly in symmetry tests. As described by the authors, "[...] when data from both Study 1 and Study 2 are combined, the 4 individuals who showed positive equivalence class outcomes also showed both sample- $\mathrm{S}^{+}$and sample- $\mathrm{S}^{-}$relations. By contrast, the single individual who had chance-level scores on equivalence tests had similar scores on tests for sample-S- relations" (Carr et al., 2000, p. 109). Explaining these differences is difficult because our subject did not show Sample-S ${ }^{+}$control but showed rejection (Sample-S control) in the test trials. What we know is that the three males from Study 1 of Carr et al. who were exposed to the blank comparison procedure during baseline responded correctly in the tests. The same strategy was insufficient to produce positive symmetry in our monkey.

With regard to the next steps in the development of this research program, we plan to (1) replicate the procedures reported herein with more monkeys, (2) further expand the rich baselines already established with our subject, Raul, by teaching further arbitrary relations with procedures designed to foster reliable select and reject control, and (3) ascertain whether developing one-to-many ARBMTS (e.g., AB, AC, $\mathrm{AD}$; now underway as reported in Campos et al., 2013) may ultimately allow the emergence of derived relations.

\section{Acknowledgements}

Funds for this research came from Edital MCT/CNPq 14/2008 Universal P481438/2008-4, Undergraduate fellowship INCT-ECCE (FINEP/ CNPq), a CNPq Research Productivity Fellowship to Olavo Galvão, and NIEHS Subaward No. 6125894/ RFS2011138 (ES15464). Manuscript preparation was supported in part by NIH Grant HD04147.

\section{References}

Alfaro, J.W.L., Silva, J.D., Jr., \& Rylands, A.B. (2012). How different are robust and gracile capuchin monkeys? An argument for the use of Sapajus and Cebus. American Journal of Primatology, 74, 273286.

Aust, U., Range, F., Steurer, M., \& Huber, L. (2008). Inferential reasoning by exclusion in pigeons, dogs, and humans. Animal Cognition, 11, 587-597.

Brino, A.L.F. (2007). Procedimentos de treino de relações condicionais em Cebus apella. [Conditional relations training procedures in Cebus apella]. Tese de doutorado. Belém: Universidade Federal do Pará. Retrieved from http://www3.ufpa.br/ppgtpc/index. php?option=com_content $\&$ view $=$ article $\&$ id $=54 \&$ Itemid $=98$

Brino, A.L., Assumpção, A.P.B., Campos, R.S., Galvão, O.F., \& McIlvane, W.J. (2010). Cebus cf. apella exhibits rapid acquisition of complex stimulus relations and emergent performance by exclusion. Psychology \& Neuroscience, 3, 209-215.

Brino, A.L.F., Galvão, O.F., \& Barros, R.S. (2009). Successive identity matching to sample tests without reinforcement in Cebus apella. Ciências \& Cognição, 14(2), 2-11.

Brino, A.L.F., Galvão, O.F., Barros, R.S., Goulart, P.R.K., \& McIlvane, W.J. (2012). Restricted stimulus control in stimulus control shaping with a capuchin monkey. Psychology \& Neuroscience, 5, 83-89.

Brino, A.L.F., Galvão, O.F., Picanço, C.R.F., Barros, R.S., Souza, C.B. A., Goulart, P.R.K., \& McIlvane, W.J. (in press). Generalized Identity Matching-to-Sample After Multiple-Exemplar Training in Capuchin Monkeys. Psychological Record, DOI 10.1007/s40732014-0035-x

Campos, R.S., Brino, A.L.F., \& Galvão, O.F. (2013). Expansão de repertório de relações arbitrárias em Sapajus sp. via exclusão. Temas em Psicologia, 21(1), 31-48.

Carr, D., Wilkinson, K.M., Blackman, D., \& Mcilvane, W.J. (2000). Equivalence classes in individuals with minimal verbal repertoires. Journal of the Experimental Analysis of Behavior, 74, 101-114.

de Rose, J.C., Hidalgo, M., \& Vasconcellos, M. (2013). Controlling relations in baseline conditional discriminations as determinants of stimulus equivalence. Psychological Record, 63, 85-98.

Frank, A.J., \& Wasserman, E.A. (2005). Associative symmetry in the pigeon after successive matching-to-sample training. Journal of the Experimental Analysis of Behavior, 84, 147-165.

Galvão, O.F., Barros, R.S., Santos, J.R., Brino, A.L.F., Brandão, S., Lavratti, C.M. ... \& McIlvane, W.J. (2005). Extent and limits of the matching concept in Cebus apella: a matter of experimental control? Psychological Record, 55, 219-232.

Johnson, C., \& Sidman, M. (1993). Conditional discriminations and equivalence relations: control by negative stimuli. Journal of the Experimental Analysis of Behavior, 33, 333-347.

Kaminski, J., Call, J., \& Fischer, J. (2004). Word learning in a domestic dog: evidence for "fast mapping." Science, 304, 1682-1683.

Kastak, C.R., Schusterman, R.J., \& Kastak, D. (2001). Equivalence classification by California sea lions using class-specific reinforcers. Journal of the Experimental Analysis of Behavior, 76, 131-158.

Lionello-DeNolf, K. (2009). The search for symmetry: 25 years in review. Learning \& Behavior, 37, 188-203.

McIlvane, W.J., \& Dube, W.V. (2003). Stimulus control topography coherence theory: foundations and extensions. Behavior Analyst, 26, 195-213.

McIlvane, W.J., Dube, W.V., Serna, R.W., Lionello-DeNolf, K.M., Barros, R.S., \& Galvão, O.F. (2011). Some current dimensions of Translational Behavior Analysis: from laboratory research to intervention for persons with autism spectrum disorders. In E.A. Mayville, \& J.A. 
Mulick (Eds.), Behavioral foundations of effective autism treatment (pp. 155-181). Cornwall-on-Hudson, NY: Sloan Publishing.

Mcllvane, W.J., Kledaras, J.B., Munson, L.C., King, K.A.J., de Rose, J.C., \& Stoddard, L.T. (1987). Controlling relations in conditional discrimination and matching by exclusion. Journal of the Experimental Analysis of Behavior, 48, 187-208.

Ray, B. A. (1969). Selective Attention: the effects of combining stimuli which control incompatible behavior. Journal of the Experimental Analysis of Behavior, 12, 539-550.

Schusterman, R.J., \& Kastak, D. (1993). A California sea lion (Zalophus californianus) is capable of forming equivalence relations. Psychological Record, 43, 823-839.

Serna, R.W., Dube, W.V., \& McIlvane, W.J. (1997). Assessing same/different judgments in individuals with severe intellectual disabilities: a status report. Research in Developmental Disabilities, 18, 343-368.

Sidman, M. (1994). Equivalence relations and behavior: a research story. Boston, MA: Authors Cooperative.
Sidman, M., Rauzin, R., Lazar, R., Cunningham, S., Tailby, W., \& Carrigan, P. (1982). A search for symmetry in the conditional discriminations of rhesus monkeys, baboons and children. Journal of the Experimental Analysis of Behavior, 37, 23-44.

Sidman, M., \& Tailby, W. (1982). Conditional discriminations vs. matching to sample: an expansion of the testing paradigm. Journal of the Experimental Analysis of Behavior, 37, 5-22.

Stromer, R., \& Osborne, J.G. (1982). Control of adolescents' arbitrary matching-to-sample by positive and negative stimulus relations. Journal of the Experimental Analysis of Behavior, 37, 329-348

Vaughan, W., Jr. (1988). Formation of equivalence sets in pigeons. Journal of Experimental Psychology: Animal Behavior Processes, 14, 36-42.

Wilkinson, K.M., Dube, W.V., \& Mcllvane, W.J. (1996). A crossdisciplinary perspective on studies of rapid word mapping in psycholinguistics and behavior analysis. Developmental Review, $16,125-148$ 